ARTICLE OPEN

\title{
Determination of triacylglycerol oxidation mechanisms in canola oil using liquid chromatography-tandem mass
} spectrometry

Shunji Kato ${ }^{1,2}$, Naoki Shimizu ${ }^{1}$, Yasuhiko Hanzawa ${ }^{1}$, Yurika Otoki ${ }^{1}$, Junya Ito ${ }^{1}$, Fumiko Kimura ${ }^{3}$, Susumu Takekoshi ${ }^{2}$, Masayoshi Sakaino ${ }^{4}$, Takashi Sano ${ }^{4}$, Takahiro Eitsuka ${ }^{1}$, Teruo Miyazawa ${ }^{5,6}$ and Kiyotaka Nakagawa ${ }^{1}$

Triacylglycerol (TG), the main component of edible oil, is oxidized by thermal- or photo- oxidation to form TG hydroperoxide $(\mathrm{TGOOH})$ as the primary oxidation product. Since $\mathrm{TGOOH}$ and its subsequent oxidation products cause not only the deterioration of oil quality but also various toxicities, preventing the oxidation of edible oils is essential. Therefore understanding oxidation mechanisms that cause the formation of $\mathrm{TGOOH}$ is necessary. Since isomeric information of lipid hydroperoxide provides insights about oil oxidation mechanisms, we focused on dioleoyl-(hydroperoxy octadecadienoyl)-TG (OO-HpODE-TG) isomers, which are the primary oxidation products of the most abundant TG molecular species (dioleoyl-linoleoyl-TG) in canola oil. To secure highly selective and sensitive analysis, authentic OO-HpODE-TG isomer references (i.e., hydroperoxide positional/geometrical isomers) were synthesized and analyzed with HPLC-MS/MS. With the use of the method, photo- or thermal- oxidized edible oils were analyzed. While dioleoyl-(10-hydroperoxy-8E,12Z-octadecadienoyl)-TG (OO-(10-HpODE)-TG) and dioleoyl-(12-hydroperoxy-9Z,13Eoctadecadienoyl)-TG (OO-(12-HpODE)-TG) were characteristically detected in photo-oxidized oils, dioleoyl-(9-hydroperoxy-10E,12Eoctadecadienoyl)-TG and dioleoyl-(13-hydroperoxy-9E,11E-octadecadienoyl)-TG were found to increase depending on temperature in thermal-oxidized oils. These results prove that our methods not only evaluate oil oxidation in levels that are unquantifiable with peroxide value, but also allows for the determination of oil oxidation mechanisms. From the analysis of marketed canola oils, photooxidized products (i.e., OO-(10-HpODE)-TG and OO-(12-HpODE)-TG) were characteristically accumulated compared to the oil analyzed immediately after production. The method described in this paper is valuable in the understanding of oil and food oxidation mechanisms, and may be applied to the development of preventive methods against food deterioration.

npj Science of Food (2018)2:1 ; doi:10.1038/s41538-017-0009-x

\section{INTRODUCTION}

Edible oil is an essential food ingredient that is mainly composed of triacylglycerol (TG), and is widely used in various food products. During industrial or culinary processes, TG oxidizes to TG hydroperoxide $(\mathrm{TGOOH})$ as the primary oxidation product by radical (e.g., auto-, thermal-) oxidation or singlet-oxygen (e.g., photo-) oxidation (Fig. 1a), ${ }^{1,2}$ and subsequently oxidized to various secondary oxidation products (e.g., aldehydes and carboxylates). ${ }^{1-3}$ The sequential formation of these oxidative products not only deteriorate the oil quality such as nutritional value, flavor and taste, but also exhibit various toxicities. ${ }^{4-8}$ Staprans et al. reported that dietary oxidized lipids are absorbed by the small intestine and distributed to lipoproteins and the liver. ${ }^{9-11}$ The circulating (and/ or accumulated) oxidized lipids are presumed to play an important role in the development of various disorders such as cardiovascular disease, ${ }^{12-14}$ Alzheimer's disease, ${ }^{15}$ and aging. ${ }^{16,17}$ Therefore, the prevention of $\mathrm{TGOOH}$ generation in oil is beneficial to our health and key to maintain the quality of edible oils. Based on these facts, considerable attention has been paid on how to analyze $\mathrm{TGOOH}$ and to determine the cause of $\mathrm{TGOOH}$ generation. ${ }^{18-21}$

Classically, TGOOH is quantified by volumetric (e.g., peroxide value $(\mathrm{POV}))^{22}$ or spectroscopic (e.g., ferrous oxidation xylenol orange $(F O X))^{23}$ methods. Of these methods, POV has been certified as an official method by the Association of Official Agricultural Chemists (AOAC), ${ }^{22}$ because of its inexpensiveness and convenience. However, since these methods are based on the oxidation-reduction reaction of iodine or ferrous, there are some drawbacks such as selectivity, sensitivity and interference of matrix compounds. To overcome such drawbacks, some selective and sensitive chromatographic methods were reported during the past few decades. ${ }^{24-30}$ For example, with the use of reverse phase (RP) chemiluminescence (CL) HPLC, we detected mono-, bis- and tris- hydroperoxides of certain TG molecular species such as trilinoleoyl-glycerol and oleoyl-dilinoleoyl-glycerol. ${ }^{25}$ Also, Alam et al. identified mono- and bis- hydroperoxides of trioleoylglycerol in olive oil using RP-HPLC coupled with electrosprayionization mass spectrometry (ESI-MS). ${ }^{28}$ However, these previous

\footnotetext{
${ }^{1}$ Food and Biodynamic Chemistry Laboratory, Graduate School of Agricultural Science, Tohoku University, Sendai, Miyagi 980-0845, Japan; ${ }^{2}$ Department of Cell Biology, Division of Host Defense Mechanism, Tokai University School of Medicine, Isehara, Kanagawa 259-1193, Japan; ${ }^{3}$ Department of Human Health and Nutrition, Shokei Gakuin University, Natori, Miyagi 981-1295, Japan; ${ }^{4}$ Fundamental Research Laboratory, J-OIL MILLS, INC., Yokohama, Kanagawa 230-0053, Japan; ${ }^{5}$ Food and Biotechnology Innovation Project, New Industry Creation Hatchery Center (NICHe), Tohoku University, Sendai, Miyagi 980-8579, Japan and ${ }^{6}$ Food and Health Science Research Unit, Graduate School of Agricultural Science, Tohoku University, Sendai, Miyagi 981-8555, Japan Correspondence: Kiyotaka Nakagawa (nkgw@m.tohoku.ac.jp)
} 


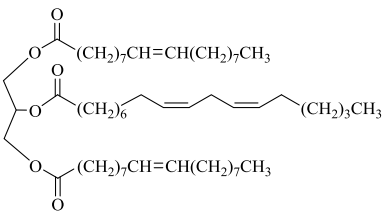

1,3-dioleoyl-2-linoleoyl-sn-glycerol*

\section{Radical oxidation products} (e.g. auto- or thermal-oxidation)

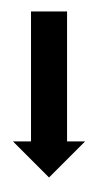

\section{Singlet-oxygen oxidation products} (e.g. photo-oxidation)
II

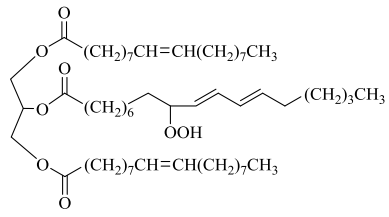

1,3-dioleoyl-2-(9-hydroperoxy$10 E, 12 E$-octadecadienoyl)-sn-glycerol (1,3-OO-2-(9-EE-HpODE)-TG)*

V

$$
\text { (1,3-OO-2-(13-EE-HpODE)-TG)* }
$$

\section{III}

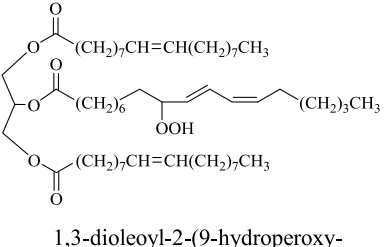

1,3-dioleoyl-2-(9-hydroperoxy$10 E, 12 Z$-octadecadienoyl)-sn-glycerol (1,3-OO-2-(9-EZ-HpODE)-TG)*

VI

$$
\begin{aligned}
& \text { 1,3-dioleoyl-2-(13-hydroperoxy- } \\
& \text { 9Z,11E-octadecadienoyl)-sn-glycerol } \\
& \text { (1,3-OO-2-(13- } Z E \text {-HpODE)-TG)* }
\end{aligned}
$$

IV

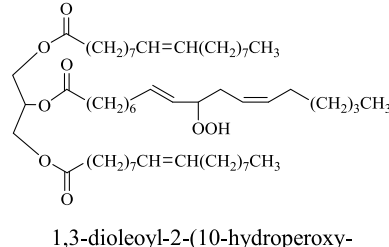

$8 E$,12Z-octadecadienoyl)-sn-glycerol (1,3-OO-2-(10-HpODE)-TG)*

VII

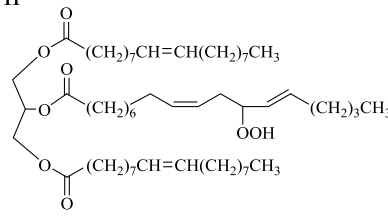

1,3-dioleoyl-2-(12-hydroperoxy9Z,13E-octadecadienoyl)-sn-glycerol (1,3-OO-2-(12-HpODE)-TG)*

*This figure illustrates $s n$-isomers bearing linoleic acid or HpODE at the $s n-2$ position.

B
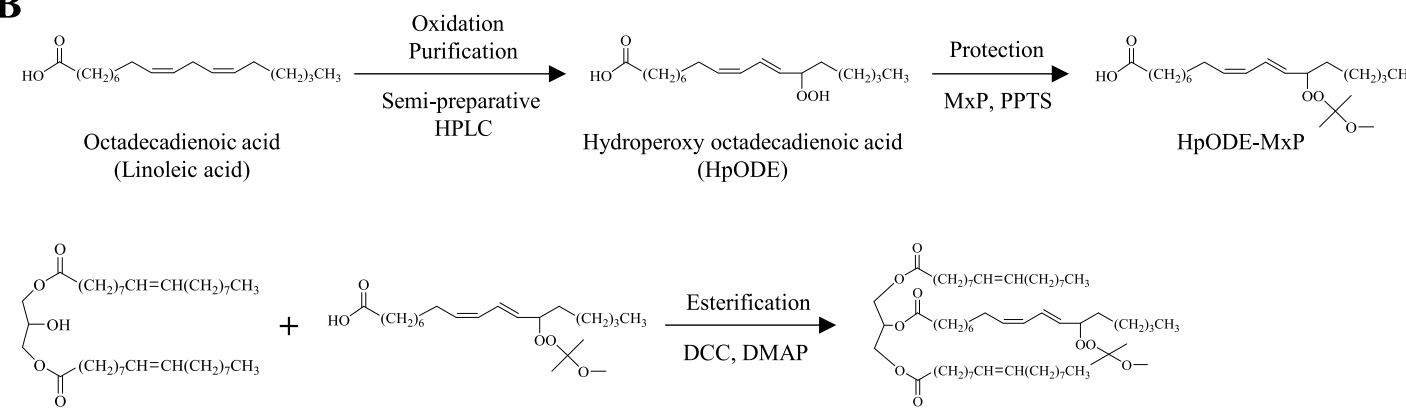

Dioleoyl glycerol

HpODE-MxP

Dioleoyl-(HpODE-MxP)-glycerol

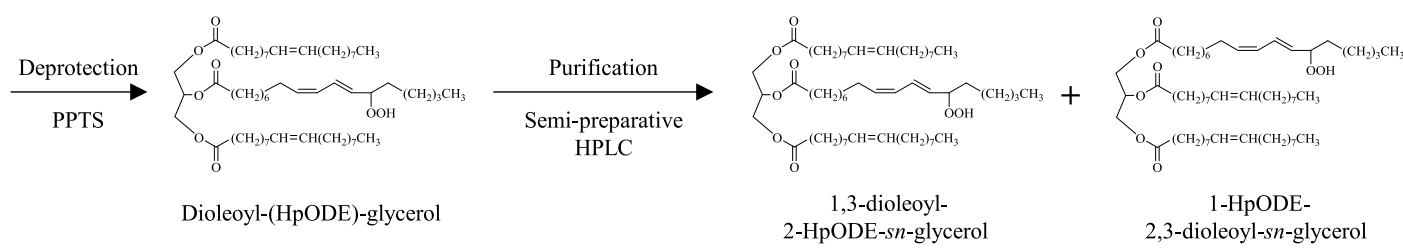

Fig. 1 Triacylglycerol (TG) oxidation mechanisms and the structures of triacylglycerol hydroperoxide isomers (a). Dioleoyl-linoleoyl glycerol (I) is oxidized to dioleoyl-(hydroperoxy octadecadienoyl) glycerol (OO-HpODE-TG, II-VII) by radical and/or singlet-oxygen oxidation. Hydroperoxide positions and geometrical structures depend on the TG peroxidation mechanisms. Schemes for the reference preparation of OO-HpODE-TG isomers (b). The references were synthesized from dioleoyl glycerol and HpODE isomers 
methods were unable to provide information about the geometrical and positional isomers of the fatty acid hydroperoxide in TG, despite the fact that these isomeric information is helpful in the elucidation of TG oxidation mechanisms (i.e., auto-, thermal- or photo-oxidation) (Fig. 1). ${ }^{1,2}$ Recently, we found that the use of alkali metals (e.g., sodium) on ESI tandem mass spectrometry (MS/ MS) enables analysis of lipid hydroperoxide positional isomers, ${ }^{31-37}$ and with the use of this technique, it was identified that the oxidation of phospholipids in mayonnaise was predominantly initiated by auto-oxidation. ${ }^{37}$

In this study, we analyzed $\mathrm{TGOOH}$ isomers in canola oil, an edible oil commonly used worldwide. The aim of this study was to analyze $\mathrm{TGOOH}$ high-sensitively, and to determine the oil oxidation mechanisms. Because the analysis of $\mathrm{TGOOH}$ is complex due to the many factors being involved (i.e., the type and position of fatty acids on the glycerol backbone, and the positional/ geometric isomerism of the hydroperoxide group), we first determined the most suitable $\mathrm{TGOOH}$ molecular species for the evaluation of canola oil oxidation mechanisms. Authentic references of the target $\mathrm{TGOOH}$ isomers were then prepared. Using the references, we quantified $\mathrm{TGOOH}$ isomers in canola oil by HPLC-ESI-MS/MS. Information on the geometrical and hydroperoxide positional isomers of $\mathrm{TGOOH}$ gave an insight into the $\mathrm{TGOOH}$ generation mechanisms in canola oil. The method described in this paper is valuable not only in the understanding of the cause of food oxidation during industrial or culinary processes, but also in developing preventive methods, including the choice of suitable anti-oxidants and packaging techniques depending on the identified oxidation mechanisms.

\section{RESULT AND DISCUSSION}

Determination of target $\mathrm{TGOOH}$ for the evaluation of oil oxidation mechanisms

Edible oil is composed of various TG molecular species. Since certain fatty acids generate multiple fatty acid hydroperoxide isomers (e.g., two types of isomers from oleic acid (OA) or six types of isomers from linoleic acid (LA)), ${ }^{1,2,33}$ it can be assumed that incalculable types of $\mathrm{TGOOH}$ molecular species/isomers are contained in oxidized edible oil. And this is the reason why the determination/quantification of edible oil oxidation is very difficult. ${ }^{29}$ Hence, we first determined the most suitable TGOOH molecular species for the evaluation of canola oil oxidation mechanisms. Based on GC analysis, it was identified that the main fatty acids that compose canola oil TG were OA $(59.7 \%)$, LA (20.0\%), and linolenic acid (LnA) (8.9\%) (Data not shown). The fatty acid combinations of TG were then determined by HPLC-MS operated in the Q1 mass- and product ion-scan mode. In the Q1 mass chromatogram, $\mathrm{m} / \mathrm{z}$ 899.8, $\mathrm{m} / \mathrm{z}$ 901.9, $\mathrm{m} / \mathrm{z}$ 903.9, $\mathrm{m} / \mathrm{z}$ 906.0, and $m / z \quad 907.9$ were abundantly detected (SI $1 \mathrm{~A})$. To analyze the fatty acid composition of these TG molecular species, product ion scan was performed (SI 1B). From the product ion analysis, we found that $\mathrm{m} / \mathrm{z} 899.8, \mathrm{~m} / \mathrm{z} 901.9$, and $\mathrm{m} / \mathrm{z} 903.9$ were composed of at least two types of TG molecular species. Collision induced dissociation (CID) of peak I (left side of $\mathrm{m} / \mathrm{z}$ 899.8) produced $\mathrm{m} / \mathrm{z}$ 620.6 and $m / z 622.8$, corresponding to the desorption of either a LA or LnA residue, respectively (SI 1 C). In consideration of monoisotopic mass ( $\mathrm{m} / \mathrm{z}$ 899.7: $\left.[\mathrm{M}+\mathrm{Na}]^{+}\right), \mathrm{m} / \mathrm{z} 899.8$ (left side) was considered to be dilinoleoyl-linolenyl glycerol. On the other hand, CID of peak II (right side of $\mathrm{m} / \mathrm{z}$ 899.8) produced $\mathrm{m} / \mathrm{z} 618.7$ and $m / z 622.8$ (SI 1D), corresponding to the desorption of either an $O A$ or LnA residue, respectively. Based on these results, the right side of $\mathrm{m} / \mathrm{z} 899.8$ was then considered to be oleoyldilinolenyl glycerol. Also, CID of peak III produced only $\mathrm{m} / \mathrm{z} 622.8$, which identified peak III as trilinoleoyl glycerol (SI 1E). Similarly, peaks IV-VIII were identified as oleoyl-linoleoyl-linolenyl glycerol (SI 1F), oleoyl-dilinoleoyl glycerol (SI 1G), dioleoyl-linolenyl glycerol
(SI 1H), dioleoyl-linoleoyl glycerol (SI 1I), and trioleoyl glycerol (SI 1J), respectively.

Of these TG, we considered that the most suitable TG molecular species for the evaluation of oxidation mechanisms are the ones that generate different isomers corresponding to different oxidation mechanisms. $\mathrm{OA}$ is hardly oxidized by thermal-oxidation, ${ }^{38}$ because thermal-oxidation (i.e., radical oxidation) is initiated by the abstraction of a hydrogen atom from the bisallylic site, which OA does not posess. ${ }^{1,2,30}$ On the other hand, because photo-oxidation (i.e., singlet oxygen-oxidation) directly occurs at double bonds by the so-called "ene" reaction, peroxidation of polyunsaturated fatty acid (PUFA) such as LnA generates excessive types of hydroperoxide isomers. ${ }^{1,2,30}$ Based on these results and facts, we considered that dioleoyl-(hydroperoxy octadecadienoyl) glycerol (OO-HpODE-TG), which possesses one molecule of LA hydroperoxide (hydroperoxy octadecadienoic acid: HpODE), is the most suitable molecular species for the evaluation of canola oil oxidation mechanisms. Because there are six types of HpODE isomers (i.e., 9-hydroperoxy10E,12Z-octadecadienoic acid: 9-EZ-HpODE, 9-hydroperoxy10E,12E-octadecadienoic acid: 9-EE-HpODE, 10-hydroperoxy$8 E, 12 Z$-octadecadienoic acid: 10-HpODE, 12-hydroperoxy-9Z,13Eoctadecadienoic acid: 12-HpODE, 13-hydroperoxy-9Z,11E-octadecadienoic acid: 13-ZE-HpODE and 13-hydroperoxy-9E,11E-octadecadienoic acid: $13-E E-H p O D E),{ }^{33}$ in this study, we analyzed each OO-HpODE-TG isomer including their sn-isomers (i.e., OO-HpODETG bearing HpODE isomers at $s n-1$ and $s n-2$ positions).

Preparation of OO-HpODE-TG isomer references

In a previous study, Alam analyzed oxidized camellia oil using HPLC-ESI-MS. ${ }^{29}$ However, the study only provided the "possible" identification of TG auto-oxidation products, because the analysis depended solely on $\mathrm{m} / \mathrm{z}$ values and did not apply any references. Since there are numerous $\mathrm{TGOOH}$ molecular species/isomers in oxidized TG, such lack of authentic references may cause fallacious analysis. In this study, to achieve highly selective analysis of $\mathrm{TGOOH}$ molecular species/isomers, we synthesized OO-HpODE-TG isomer references from diacylglycerol and six HpODE isomers (Fig. 1b). Since it is difficult to chromatographically separate esterified HpODE isomers, ${ }^{31}$ the six HpODE isomers were first separated and collected in its free form, and subsequently each esterified with dioleoyl glycerol. ${ }^{39}$ Because we used a mixture of dioleoyl glycerol isomers (i.e., 1,3-dioleoyl-2-hydroxy-sn-glycerol and 1-hydroxy-2,3-dioleoyl-sn-glycerol), the obtained OO-HpODETG was a mixture of 1,3-dioleoyl-2-(HpODE)-sn-glycerol (1,3-OO-2(HpODE)-TG) and 1-(HpODE)-2,3-dioleoyl-sn-glycerol (1-(HpODE)2,3-OO-TG). But, even when pure 1,2-dioleoyl-3-hydroxy-snglycerol is employed, the resultant OO-HpODE-TG is known to be a mixture of the isomers, because 1,2-diacyl glycerol isomerizes to 1,3-diacyl glycerol which is thermodynamically more stable. ${ }^{40}$ Hence, to avoid this isomerization reaction, in this study, OOHpODE-TG $s n$-isomers were separated after esterification. In order to determine the HpODE binding $s n$-position of the obtained OOHpODE-TG, a portion of the references were catalyzed by a-lipase, which act specifically at the $s n-1$ and $s n-3$ positions of triacylglycerol. Before this reaction, the hydroperoxide group of OO-HpODE-TG was protected with 2-methoxypropene $(\mathrm{MxP})^{41}$ to avoid the partial decomposition of the hydroperoxide group by lipase. ${ }^{42}$ From the HPLC-MS analysis of the hydrolysate, we identified specific monoacylglycerols depending on the HpODE binding positions on the glycerol backbone (i.e., $\mathrm{m} / \mathrm{z} 481.3$ generated from 1,3-OO-2-(HpODE)-TG or $\mathrm{m} / \mathrm{z} 379.3$ generated from 1-(HpODE)-2,3-OO-TG) (SI 2). Based on these results, we determined the identities of the 12 OO-HpODE-TG references, and further used the references for MS/MS and HPLC-MS/MS analysis. 
A 1,3-OO-2-(9-E,E-HPODE)-TG

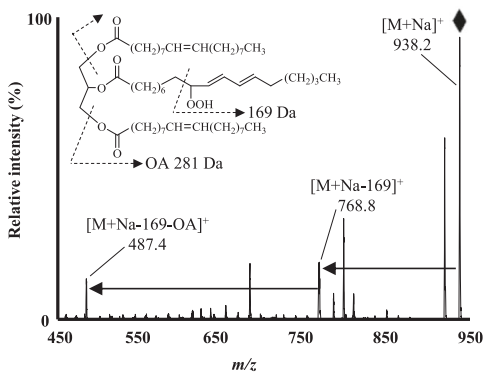

D 1,3-OO-2-(13-E,E-HpODE)-TG

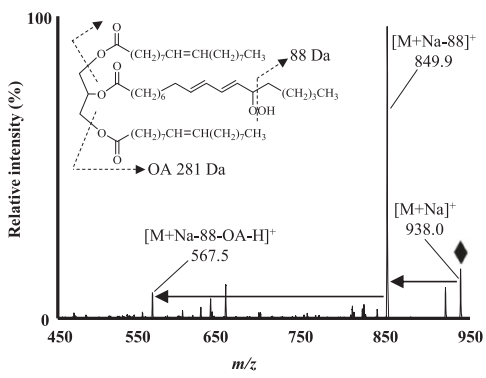

G 1-(9-E,E-HpODE)-2,3-OO-TG

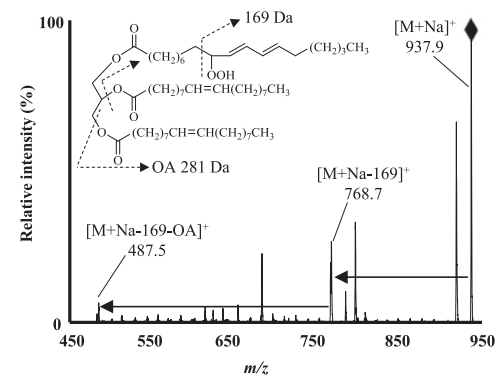

J 1-(13-E,E-HpODE)-2,3-OO-TG

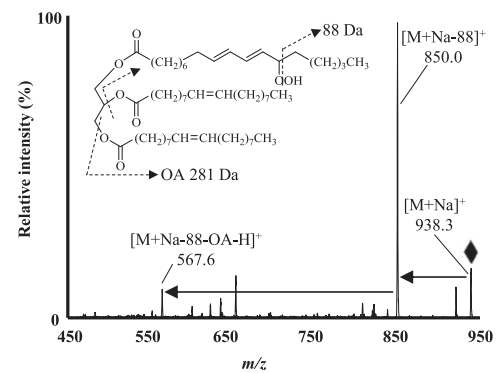

В 1,3-OO-2-(9-E,Z-HpODE)-TG

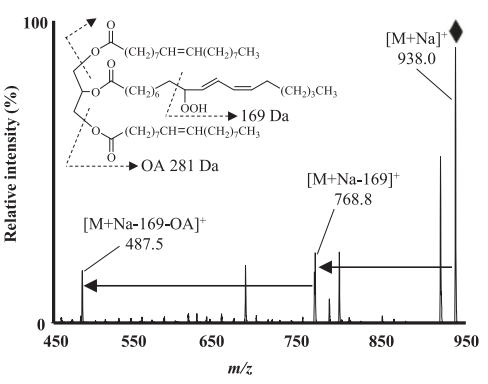

E 1,3-OO-2-(13-Z,E-HpODE)-TG

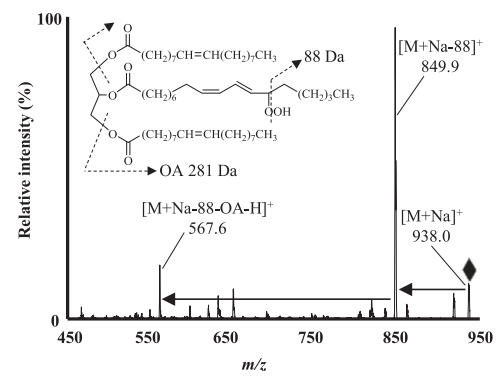

H 1-(9-E,Z-HpODE)-2,3-OO-TG

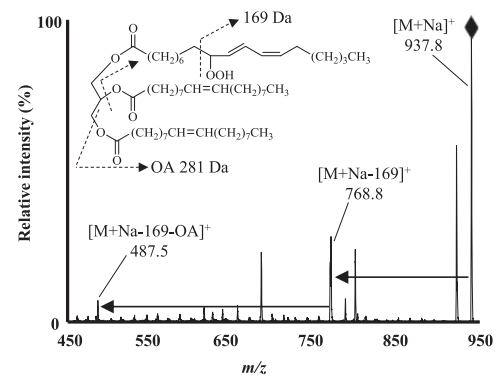

K 1-(13-Z,E-HpODE)-2,3-OO-TG

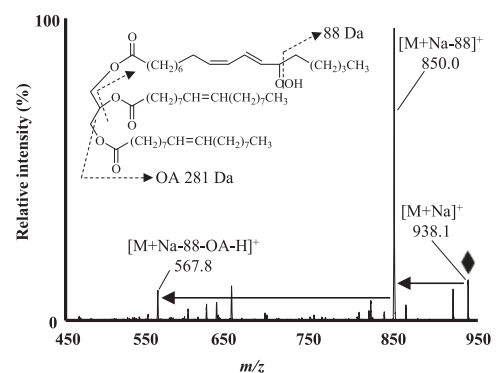

C 1,3-OO-2-(10-HpODE)-TG

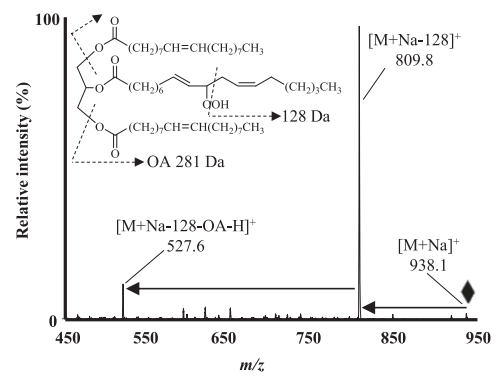

F 1,3-OO-2-(12-HpODE)-TG

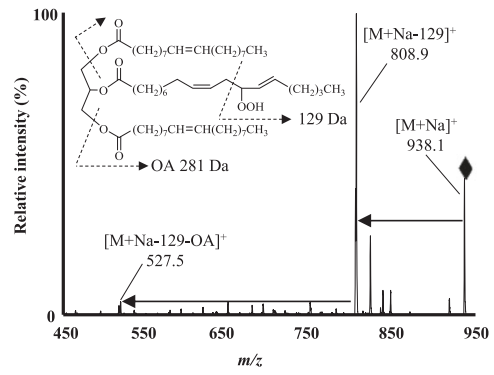

I 1-(10-HpODE)-2,3-OO-TG

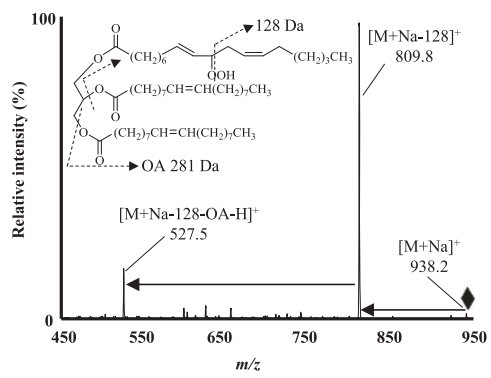

L 1-(12-HpODE)-2,3-OO-TG

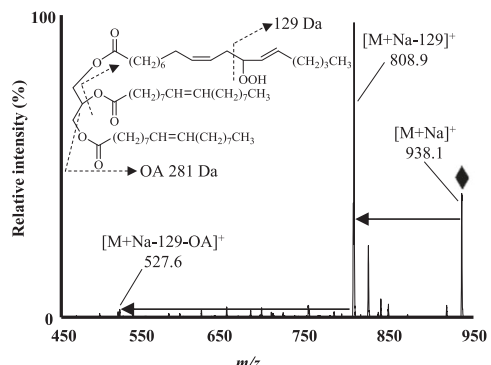

Fig. 2 The product ion mass spectra of reference OO-HpODE-TG isomers (A-L). Reference OO-HpODE-TG isomers were dissolved in methanol containing $0.1 \mathrm{mM}$ sodium acetate $(1 \mu \mathrm{M})$ and infused directly into the $\mathrm{MS} / \mathrm{MS}$ system at a flow rate of $10 \mu \mathrm{L} / \mathrm{min}$. Insets show the speculated fragmentation patterns of OO-HpODE-TG isomers

MS/MS and HPLC-MS/MS analysis of OO-HPODE-TG isomers As described in the Introduction, the isomerism of HpODE (i.e., hydroperoxide positional and cis-trans structural isomers) is essential to evaluate oxidation mechanisms in edible oil. ${ }^{1,2,31,33,34,37}$ In this study, we aimed to discriminate these isomers using MS/MS and HPLC separation techniques. Because the selective analysis of HpODE isomers were achieved in the presence of sodium in previous studies, ${ }^{31-37}$ OO-HpODE-TG references were dissolved in solvents containing sodium acetate. The references were analyzed by ESI operated in the positive ion mode. In each Q1 mass spectrum of the synthesized OO-HpODE-
TG isomers, $\mathrm{m} / \mathrm{z} 938.2$ corresponding to $[\mathrm{M}+\mathrm{Na}]^{+}$was clearly detected (SI 3). Product ion analysis of these $\mathrm{m} / \mathrm{z} 938.2$ each gave a unique neutral loss (i.e., $169 \mathrm{Da}$ for isomers bearing 9-HpODE (Fig. 2a, b, g and h), $128 \mathrm{Da}$ for isomers bearing 10-HpODE (Fig. 2c, i), $129 \mathrm{Da}$ for isomers bearing $12-\mathrm{HpODE}$ (Fig. 2f, I) and $88 \mathrm{Da}$ for isomers bearing 13-HpODE (Fig. 2d, e, j and k)). No significant difference in the product ion mass spectrum was observed between the OO-HpODE-TG geometrical isomers. Multiple reaction monitoring (MRM) pairs for HPLC-MS/MS analysis were selected based on the observed product ions (i.e., $938.0>768.8$ for isomers bearing $9-\mathrm{HpODE}, 938.0>809.8$ for isomers bearing 
10-HpODE, $938.0>808.8$ for isomers bearing 12-HpODE and 938.0 $>849.8$ for isomers bearing $13-\mathrm{HpODE}$ ).

Subsequently, the geometrical isomerism of OO-HpODE-TG isomers were analyzed by HPLC-MS/MS. In this study, we used silica-based normal phase- (NP-) HPLC for the separation of OOHpODE-TG isomers, because NP-HPLC is generally effective for the
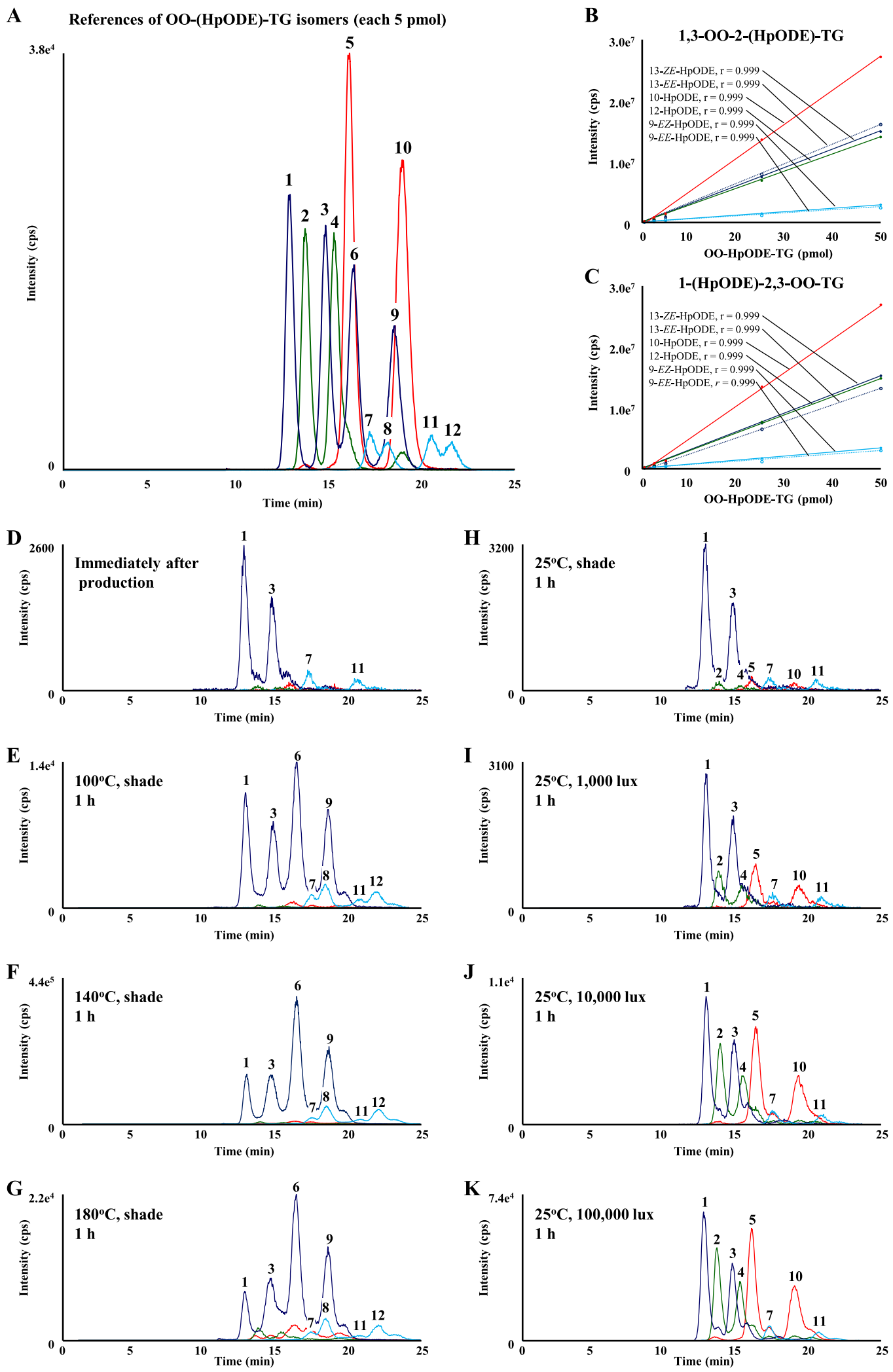
Fig. 3 MRM chromatograms of reference OO-HpODE-TG isomers (a). OO-HpODE-TG isomer references (5 pmol each) were analyzed with MRM $(\mathrm{m} / \mathrm{z} 938.0>768.8$ for isomers bearing $9-\mathrm{HpODE}, \mathrm{m} / \mathrm{z} 938.0>809.8$ for isomers bearing $10-\mathrm{HpODE}, \mathrm{m} / \mathrm{z} 938.0>808.8$ for isomers bearing $12-$ HpODE and $\mathrm{m} / \mathrm{z}$ 938.0 > 849.8 for isomers bearing 13-HpODE). Peaks 1-12 and their retention time were as follows: (1) 1,3-OO-2-(13-ZEHpODE)-TG (12.6 min); (2) 1,3-OO-2-(12-HpODE)-TG (13.4 min); (3) 1-(13-ZE-HpODE)-2,3-OO-TG (14.5 min); (4) 1-(12-HpODE)-2,3-OO-TG (15.0 $\mathrm{min})$; (5) 1,3-OO-2-(10-HpODE)-TG (15.9 min); (6) 1,3-OO-2-(13-EE-HpODE)-TG (16.1 min); (7) 1,3-OO-2-(9-EZ-HpODE)-TG (17.0 min); (8) 1,3-OO-2(9-EE-HpODE)-TG (18.0 min); (9) 1-(13-EE-HpODE)-2,3-OO-TG (18.4 min); (10) 1-(10-HpODE)-2,3-OO-TG (18.8 min); (11) 1-(9-EZ-HpODE)-2,3-OOTG (20.5 min); (12) 1-(9-EE-HpODE)-2,3-OO-TG (21.6 min). Calibration curves of reference OO-HpODE-TG isomers (1,3-OO-2-(HpODE)-TG (b) and 1-(HpODE)-2,3-OO-TG (c)). Different amounts of OO-HpODE-TG isomers (0.25-50 pmol) were analyzed by optimized LC-MS/MS MRM. MRM chromatograms of thermal- $(\mathbf{d}-\mathbf{g})$ and photo- $(\mathbf{h}-\mathbf{k})$ oxidized oil. Oxidized oils were 100 -fold diluted with hexane, and a portion (50 $\mu \mathrm{L})$ was analyzed using LC-MS/MS MRM. Peak numbers refer to the OO-HpODE-TG isomers described above

promote ionization. With the use of these MS/MS and HPLC techniques, we analyzed the 12 OO-HpODE-TG references. Under optimized HPLC-MS/MS conditions, all isomers were clearly detected (Fig. 3a). The retention time for each isomer is shown in Fig. 3a. For all isomers, calibration curves demonstrated good linearity within the range of $0.25-50 \mathrm{pmol} /$ injection (Fig. $3 \mathrm{~b}, \mathrm{c}$ ).

Analysis of thermal- or photo-oxidized canola oil and the determination of oil oxidation mechanisms

In this study, we analyzed fresh canola oil (i.e., canola oil collected immediately after production) and oxidized oil using authentic references and optimized HPLC-MS/MS conditions. Fresh canola oil was oxidized by either thermal- $\left(25-180^{\circ} \mathrm{C}\right.$, shade) or photo$\left(25^{\circ} \mathrm{C}, 1000-100,000\right.$ lux: corresponding to office lighting-direct sunlight) oxidation for $2 \mathrm{~h}$, assuming that edible oil would be oxidized under common use or storage.

The typical chromatograms of fresh, thermal-oxidized and photo-oxidized canola oil are shown in Fig. 3d-k. In the chromatograms of oxidized oil, some unidentified peaks were detected near the 1,3-OO-2-(HpODE)-TG and 1-(HpODE)-2,3-OOTG. This suggests the possibility that other $\mathrm{TGOOH}$ molecular species were co-eluted with 1,3-OO-2-(HpODE)-TG and 1-(HpODE)2,3-OO-TG. In this study, to secure highly selective analysis, HPLC-MS/MS/MS analysis was further performed (Fig. 4). For the MS/MS/MS analysis of OO-(9-HpODE)-TG, we used $\mathrm{m} / \mathrm{z} 768.8$ as a second precursor ion. As mentioned above, $\mathrm{m} / \mathrm{z} 768.8$ is generated from HpODE bearing hydroperoxide group at the ninth position (Fig. 2a, b, g and h). We then analyzed product ions generated from $m / z 768.8$, to check the other fatty acids type/combination (sn-1 and $s n-3$ or $s n-2$ and $s n-3)$. Total ion current (TIC) chromatogram and mass spectra of the product ion generated from $\mathrm{m} / \mathrm{z} 768.8$ (photo-oxidized oil (10,000 lux)) are shown in Fig. 4a. On peaks I and II, fragmentation of $\mathrm{m} / \mathrm{z} 768.8$ predominantly generated the ion $\mathrm{m} / \mathrm{z} 487.5$ corresponding to the dissociation of an OA residue $(281.3 \mathrm{Da})$. These results were also observed in the product ion mass spectra of OO-HpODE-TG isomers (Fig. 2). This result indicates that peaks I and II are composed of OO-(9-HpODE)-TG. Similarly, we analyzed OO-(10HpODE)-TG in photo oxidized oil (10,000 lux) (Fig. 4b). On peaks I and III, fragmentation of $\mathrm{m} / \mathrm{z} 809.8$ predominantly generated the ion $\mathrm{m} / \mathrm{z} 527.4$ corresponding to the dissociation of the oleoyl residue and a proton (282 Da) (Figs. $2 \mathrm{c}$, i and 4 b). On the other hand, we found that peaks II and IV generated $\mathrm{m} / \mathrm{z} 525.4$ or 529.4 corresponding to the dissociation of a stealoyl or a linoleoyl residue, respectively. This suggests that peaks II and IV are isomers of OO-(9-HpODE)-TG (M.W. 915) such as stealoyl-linoleoyl-(13HpODE)-TG (M.W. 915). The same MS/MS/MS analysis were performed on other isomers (Fig. 4c, d) as well as thermaloxidized oil (Data not shown). These results prove that the targeted MRM peaks are definitely OO-(HpODE)-TG.

We then calculated the peak areas of OO-HpODE-TG isomers in fresh canola oil from MRM chromatograms. We found that OO-(9EZ-HpODE)-TG and OO-(13-ZE-HpODE)-TG were present despite being analyzed immediately after production (Fig. 5, 0 min). When the oil was oxidized at $25^{\circ} \mathrm{C}$, OO-(13-ZE-HpODE)-TG tended to increase, while other OO-HpODE-TG isomers did not increase (Fig. 5a, b). On the other hand, when the oil was oxidized at high temperatures $\left(100-180^{\circ} \mathrm{C}\right)$, not only OO-(13-ZE-HpODE)-TG but also OO-(9-EZ-HpODE)-TG, OO-(13-EE-HpODE)-TG, and OO-(9-EEHPODE)-TG significantly increased (Fig. 5c-h). Of these OOHpODE-TG isomers, OO-(13-EE-HpODE)-TG and OO-(9-EEHpODE)-TG were found to increase greater than OO-(13-ZEHPODE)-TG and OO-(9-EZ-HpODE)-TG, even if TGOOH was decomposed by high temperature $\left(180^{\circ} \mathrm{C}\right)$. In contrast, in photooxidized oil, OO-(9-EE-HpODE)-TG and OO-(13-EE-HpODE)-TG were not detected at all illumination intensities (Fig. 5i-n). Also, it was found that OO-(12-HpODE)-TG and OO-(10-HpODE)-TG increased depending on the exposure time and illumination intensity. Detailed values and POV for each sample are shown in SI 4.

In the case of free fatty acid peroxidation, theoretically, 9HpODE and 13-HpODE (or 10-HpODE and 12-HpODE) are equally generated by radical- or singlet oxygen-oxidation. ${ }^{1}$ However, in the present study concerning TG, the concentration of OO-(12HpODE)-TG and OO-(13-ZE-HpODE)-TG were higher than that OO-(10-HpODE)-TG and OO-(9-EZ-HpODE)-TG, respectively (Fig. 5). Moreover, in photo-oxidized oil, we found that the concentration of OO-(12-HpODE)-TG exceeded that of OO-(9-EZ-HpODE)-TG, despite the fact that OO-(9-EZ-HpODE)-TG is generated by both radical- and singlet oxygen-oxidation. ${ }^{1}$ These results suggest that steric hindrance is involved in TG peroxidation. Alam ${ }^{29}$ and Neff et al. $^{26}$ reported that there are no $s n$-preference during the oxidation of trilinoleoyl-glycerol. On the other hand, other studies have reported that the fatty acid attached to the $s n-2$ position is tolerant to oxidation than the fatty acid attached to the $s n-1$ ( $s n-3)$ position. ${ }^{44,45}$ Further study would be required to elucidate steric hindrance, such as sn-position, fatty acid type and/or hydroperoxide position, during TG peroxidation.

In this study, we reliably identified OO-HpODE-TG molecular species/isomers peaks using MS/MS (MS/MS/MS) analysis and authentic references, although the analysis of TGOOH (even intact TG) is very difficult due to the various types of fatty acids and their combinations. ${ }^{29}$ The novel methods demonstrated that OO-(10HPODE)-TG and OO-(12-HpODE)-TG were characteristically detected in photo-oxidized oil, and OO-(13-EE-HpODE)-TG and OO-(9-EE-HpODE)-TG increased depending on the temperature during thermal-oxidation. Our methods evaluated oil oxidation in levels that are unquantifiable with POV (even the oil oxidized by 1000 lux irradiation for $10 \mathrm{~min}$ ).

Determination of oxidation mechanisms of marketed canola oil Lastly, to evaluate the oxidation mechanisms during the transportation or storage of edible oils, we analyzed marketed canola oil products purchased from four different local retailers (Fig. 6). The analyzed marketed canola oils were the same product as the fresh oil used above. The concentration of the total OOHPODE-TG was 10-25 times higher in marketed oil compared to the fresh oil. Importantly, photo-oxidation products (i.e., OO-(12HpODE)-TG and OO-(10-HpODE)-TG) were clearly detected in all marketed canola oils, although these isomers were not detected in the fresh oil. Furthermore, high temperature-oxidation products 


\section{A}

\section{OO-(9-HpODE)-TG}

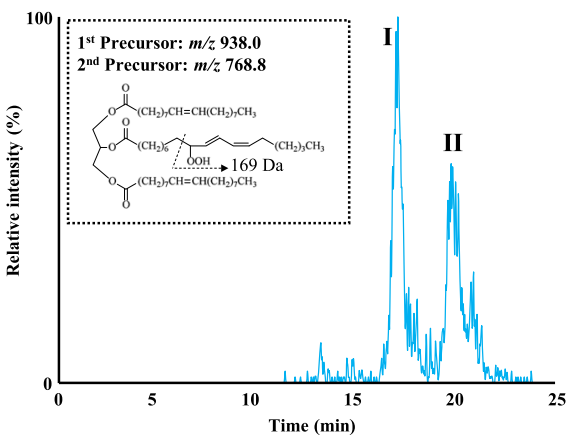

B

OO-(10-HpODE)-TG
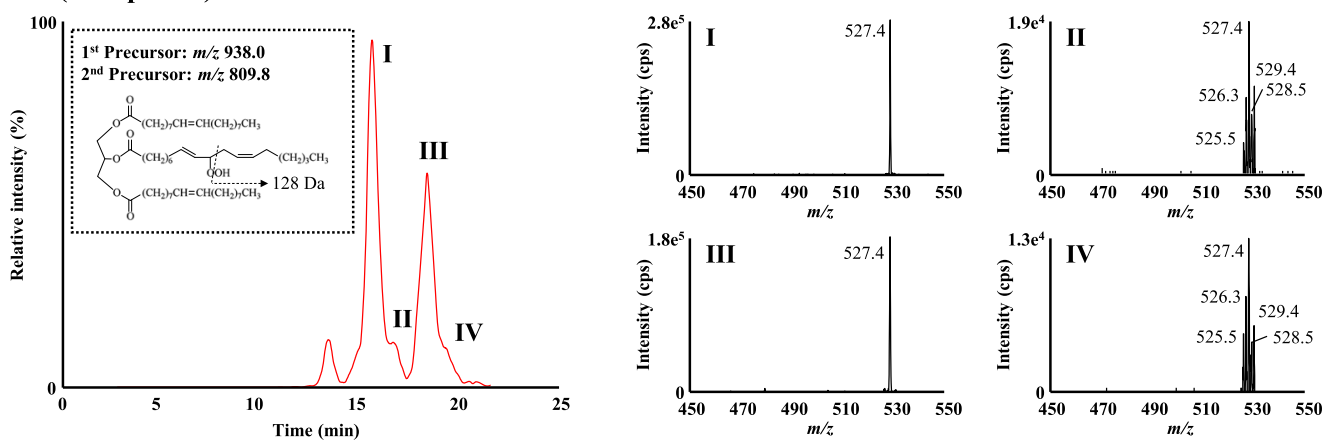

C
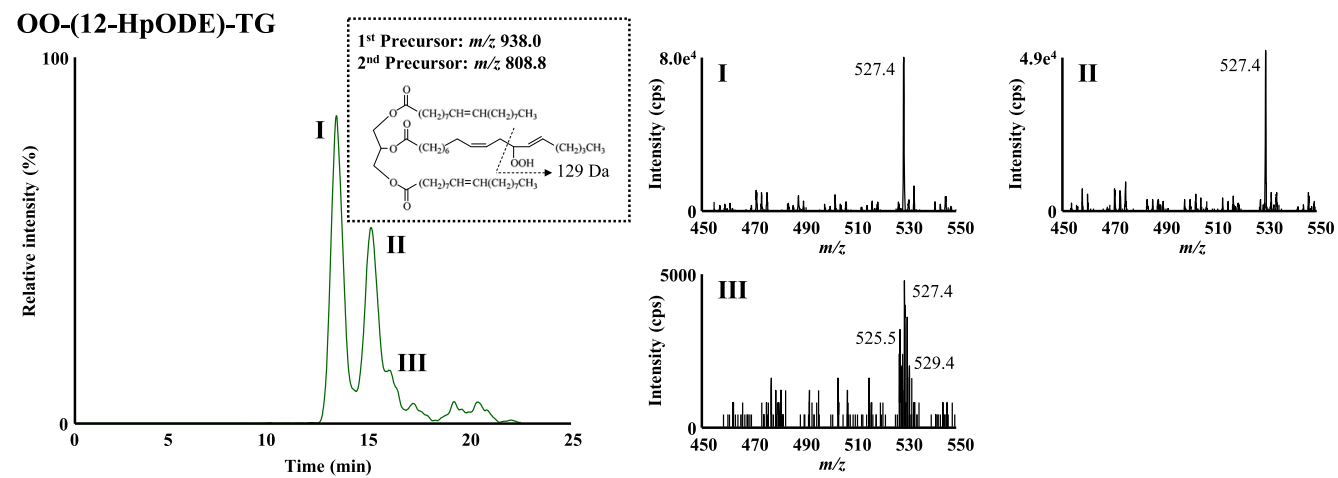

D

OO-(13-HpODE)-TG
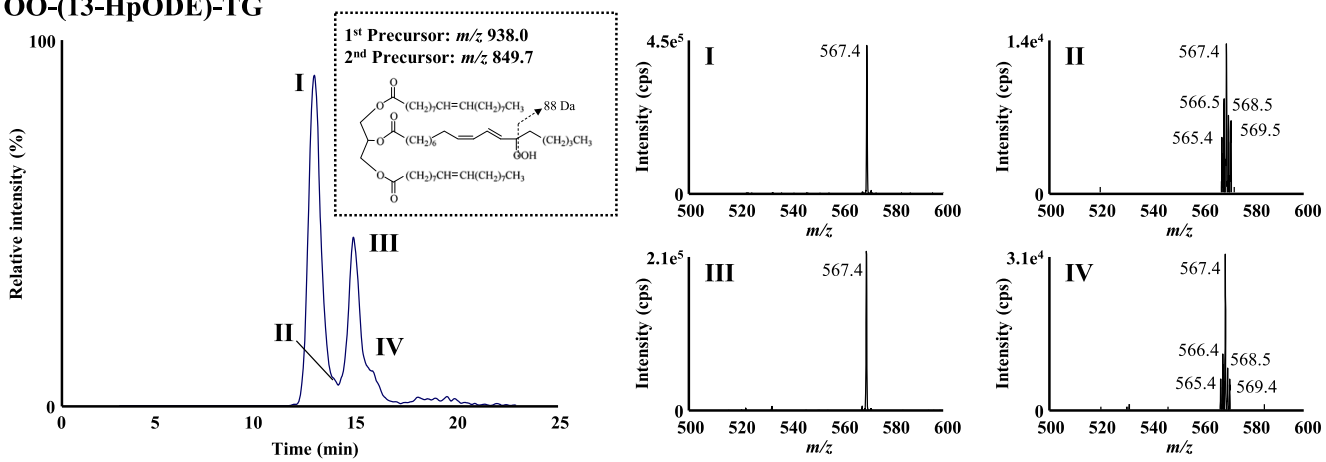

Fig. 4 MS/MS/MS analysis of photo-oxidized oil. The ion $\mathrm{m} / \mathrm{z} 938.0$ was selected as a first precursor ion, and $\mathrm{m} / \mathrm{z}$ 768.8 (OO-(9-HpODE)-TG (a); $\mathrm{m} / \mathrm{z} 809.8$ (OO-(10-HpODE)-TG (b); $\mathrm{m} / \mathrm{z} 808.8$ (OO-(12-HpODE)-TG (c); and m/z 849.7 (OO-(13-HpODE)-TG (d); were selected as a second precursor ion. Each chromatogram is a total ion current chromatogram of the product ion generated from the second precursor ion. MS/MS/ MS spectra (I-IV) demonstrating product ions generated from the second precursor ion. Photo-oxidized oil were 100-fold diluted with hexane, and a portion $(50 \mu \mathrm{L})$ was analyzed under optimized LC-MS/MS/MS conditions 


\section{Thermal-oxidation}

1,3-OO-2-(HpODE)-TG

A

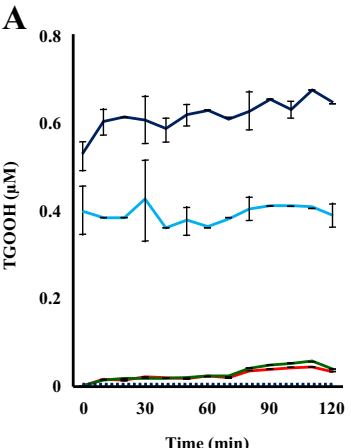

C

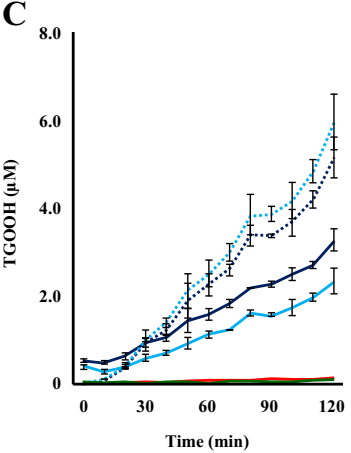

E

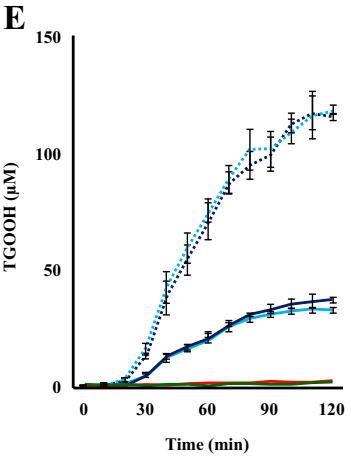

G

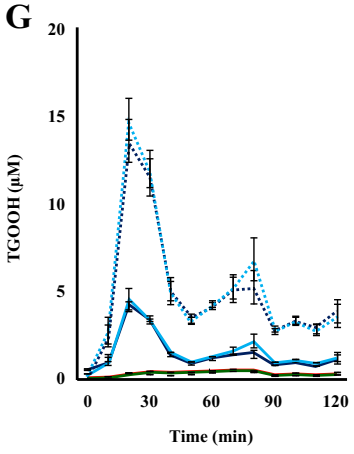

1-(HpODE)-2,3-OO-TG

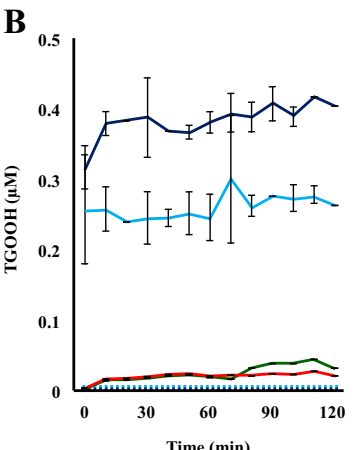

D

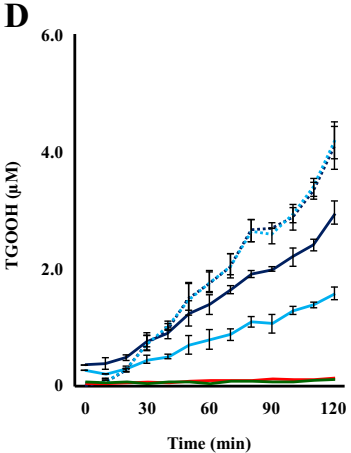

F

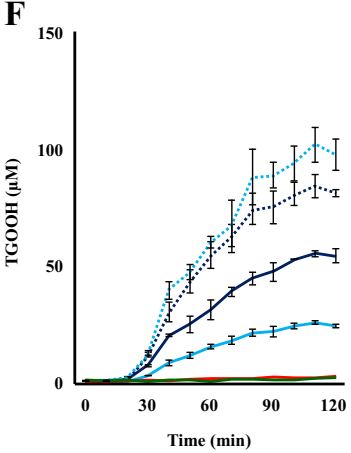

H

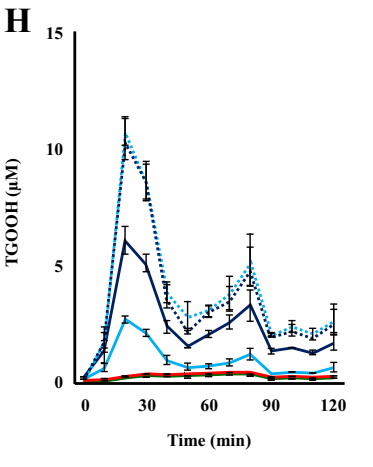

Photo-oxidation

1,3-OO-2-(HpODE)-TG

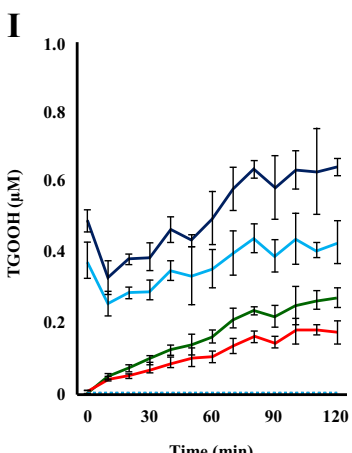

1-(HPODE)-2,3-OO-TG
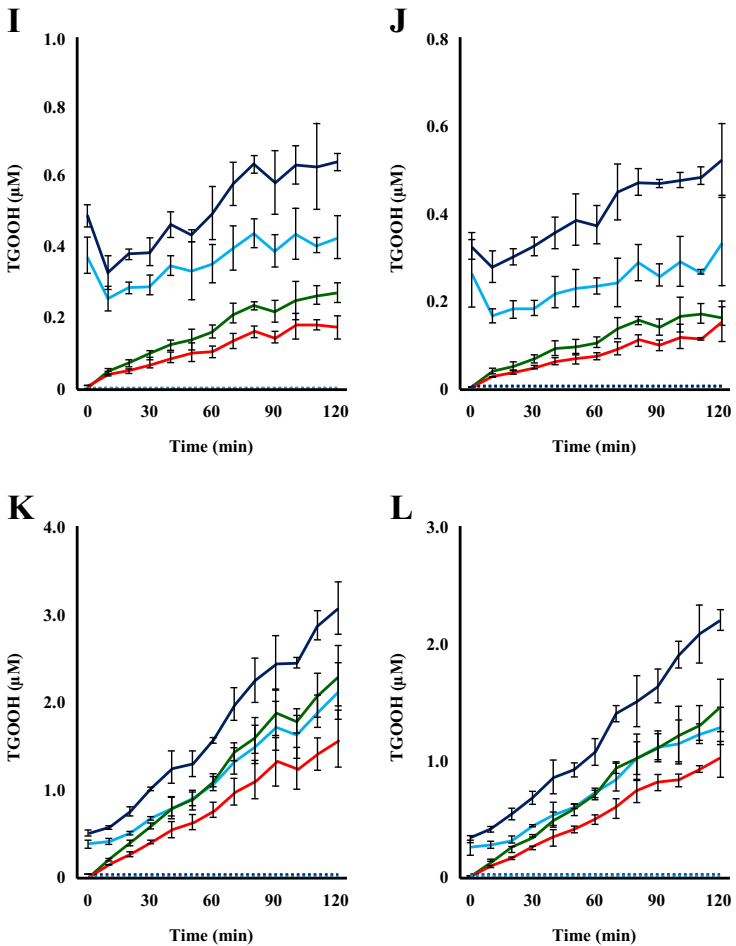

L

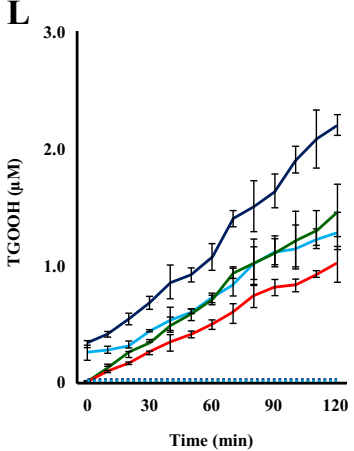

M

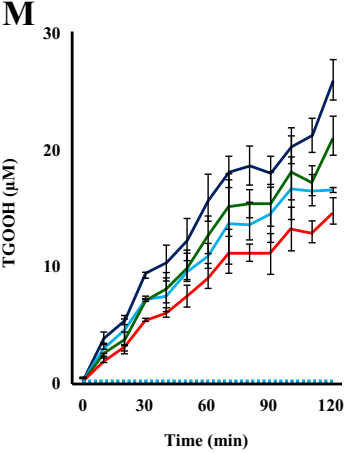

$\mathbf{N}$

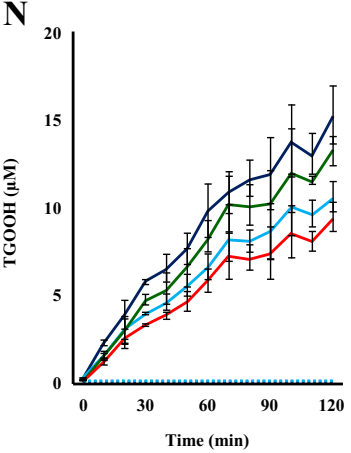

OO-(9-EZ-HpODE)-TG

OO-(9-EE-HpODE)-TG

OO-(10-HрODE)-TG

OO-(12-HpODE)-TG

\section{OO-(13-ZE-HpODE)-TG}

OO-(13-EE-HPODE)-TG

Fig. 5 Concentrations of 1,3-OO-2-(HpODE)-TG and 1-(HpODE)-2,3-OO-TG isomers in oxidized oil. Fresh oil was oxidized under the following conditions: $25^{\circ} \mathrm{C}$, shade $(\mathbf{a}, \mathbf{b}) ; 100^{\circ} \mathrm{C}$, shade $(\mathbf{c}, \mathbf{d}) ; 140^{\circ} \mathrm{C}$, shade $(\mathbf{e}, \mathbf{f}) ; 180^{\circ} \mathrm{C}$, shade $(\mathbf{g}, \mathbf{h}) ; 25^{\circ} \mathrm{C}, 1000$ lux (i, j); $25^{\circ} \mathrm{C}, 10,000$ lux $(\mathbf{k}, \mathbf{I}) ; 25^{\circ} \mathrm{C}$, $100,000$ lux $(\mathbf{m}, \mathbf{n})$. Oxidized oils were 100-fold diluted with hexane, and a portion (50 $\mu \mathrm{L})$ was analyzed under optimized LC-MS/MS MRM conditions 
A 1,3-OO-2-(HPODE)-TG

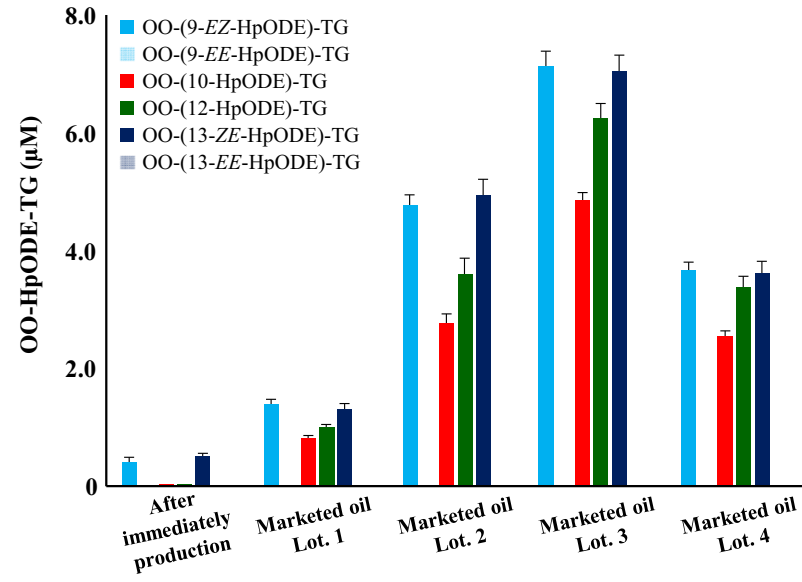

B

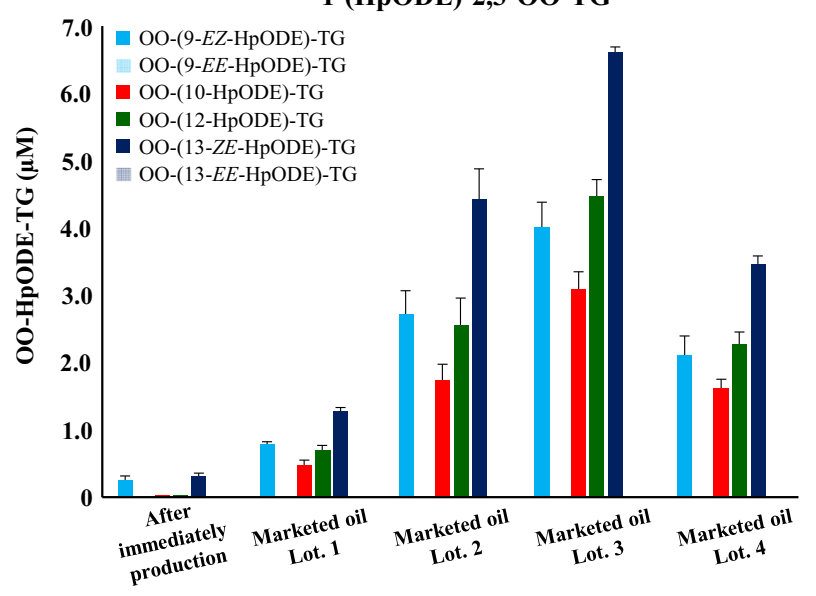

Fig. 6 (A and B) Concentrations of OO-HPODE-TG isomers in marketed canola oil. Marketed oils were analyzed immediately after opening. Oils were 100 -fold diluted with hexane and a portion $(50 \mu \mathrm{L})$ was analyzed under optimized LC-MS/MS MRM conditions

(i.e., OO-(13-EE-HpODE)-TG and OO-(9-EE-HpODE)-TG) were not detected in any canola oils. There was no difference in the isomer composition ratio between the marketed oils, despite the fact that the oil used in this study was purchased from different retailers. These results suggest that the oxidation of marketed canola oil is initiated by photo-oxidation around room temperature. Also, it can be assumed that canola oil is susceptible to photo-oxidation rather than radical oxidation. Generally, radical trapping reagents such as tocopherol is contained (and/or added) in commercial edible oils. Therefore, it can be assumed that radical oxidation is not enhanced at least before unsealing. On the other hand, since photo-oxidation products were accumulated in significant amounts, addition of singlet oxygen trapping reagents such as carotenoids ${ }^{46-49}$ and improvement of packaging techniques (e.g., use of brown bottle) may prolong the shelf life of edible oils.

Taken together, isomeric information of lipid hydroperoxides (i.e., geometrical and hydroperoxide-positional isomers) can be used to elucidate lipid oxidation mechanisms (thermal- and photo-oxidation). In this study, $\mathrm{TGOOH}$ isomers in canola oil were analyzed by preparing authentic isomer references and performing LC-MS/MS (MS/MS/MS) analysis. We demonstrated that the oxidation of marketed canola oil is predominantly caused by photo-oxidation. To the best of our knowledge, this is the first report that analyzed $\mathrm{TGOOH}$ at the molecular species and hydroperoxide positional-/geometrical-isomer levels. The method described in this paper would be valuable in the understanding of oil or food peroxidation mechanisms, and the development of preventive methods against food deterioration.

\section{MATERIALS AND METHODS}

Materials

Dioleoyl glycerol (mixture of 1,2-dioleoyl-3-hydroxy-sn-glycerol and 1,3dioleoyl-2-hydroxy-sn-glycerol), LA, heptadecanoic acid, butyl hydroxy toluene (BHT), rose bengal, MxP, $N, N^{\prime}$-dicyclohexylcarbodiimide (DCC) and dimethylaminopyridine (DMAP) were purchased from Wako Pure Chemical Industries, Ltd. (Osaka, Japan). Pyridinium $p$-toluenesulfonate (PPTS) was obtained from Sigma-Aldrich (St. Louis, MO, USA). Fresh canola oil was collected immediately after production at J-OIL MILLS, Inc. (Tokyo, Japan), and was stored under nitrogen gas and shading until use. Marketed canola oil products were purchased at four different local market in Sendai Japan. a-Lipase (Lipase F3G) was kindly provided from Amano Enzyme Inc. (Aichi, Japan). All other reagents were of the highest grade available.

Determination of target TGOOH molecular species of canola oil The fatty acid composition of TG in canola oil was analyzed by GC. Fresh canola oil $(5 \mathrm{mg})$ was mixed with an internal standard $(50 \mu \mathrm{g}$ of heptadecanoic acid) in a screw cap test tube. Then, $2 \mathrm{~mL}$ of methanolic hydrogen chloride $(5 \%, v / v)$ with $0.2 \mathrm{~mL}$ of benzene and $2 \mu \mathrm{L}$ of BHT methanolic solution $(0.01 \%$, wt $\%)$ were added. The sample was methyl esterified at $100^{\circ} \mathrm{C}$ under a nitrogen atmosphere. After $1 \mathrm{~h}, 5 \mathrm{~mL}$ of aqueous potassium carbonate $(6 \%$, wt $\%$ ) and $1 \mathrm{~mL}$ of hexane were added. The mixture was partitioned by centrifugation $\left(1000 \times g\right.$ for $5 \mathrm{~min}$ at $\left.4^{\circ} \mathrm{C}\right)$ into two layers. The upper hexane layer was collected, and remaining aqueous layer was re-extracted with $1 \mathrm{~mL}$ of hexane. The combined hexane layer was evaporated under a nitrogen gas stream, and the residue was dissolved in $1 \mathrm{~mL}$ of hexane. A $2 \mu \mathrm{L}$ aliquot was analyzed with GC-4000 (GL Sciences Inc., Tokyo, Japan) equipped with a DB-225 column (length, $30 \mathrm{~m}$; internal diameter, $0.32 \mathrm{~mm}$; film thickness, $0.25 \mu \mathrm{m}$; Agilent Technologies, Santa Clara, CA, USA). Helium gas was used as the mobile phase. The injector and detector temperatures were set at 220 and $250{ }^{\circ} \mathrm{C}$ respectively. The gradient profile was as follows: $140-180^{\circ} \mathrm{C}\left(8^{\circ} \mathrm{C} / \mathrm{min}\right.$ linear), $180-220^{\circ} \mathrm{C}\left(3^{\circ} \mathrm{C} / \mathrm{min}\right.$ linear), and $220^{\circ} \mathrm{C}$ (for $25 \mathrm{~min}$ ).

TG molecular species were analyzed by $\mathrm{Q} 1$ mass- and product ion-scan mode using LC-MS/MS. A Prominence liquid chromatography system (Shimadzu, Kyoto, Japan) was equipped with a 4000 QTRAP mass spectrometer (SCIEX, Tokyo, Japan). Fresh canola oil was 10,000-fold diluted with methanol/2-propanol (100:1, v/v) and analyzed using an ODS column ( $5 \mathrm{C}_{18}$-MS-II, $5 \mu \mathrm{m}, 4.6 \times 250 \mathrm{~mm}$, nacalai tesque, Kyoto, Japan) with a binary gradient consisting of solvent $A$ (methanol containing $0.1 \mathrm{mM}$ sodium acetate) and solvent $B$ (2-propanol containing $0.1 \mathrm{mM}$ sodium acetate). The gradient profile was as follows: $0-20 \mathrm{~min}, 50-70 \%$ B linear. The flow rate was $1.0 \mathrm{~mL} / \mathrm{min}$, and the column temperature was $40^{\circ} \mathrm{C}$. Elution was split at the post-column and one of the split eluents was sent to the MS/MS system at $0.2 \mathrm{~mL} / \mathrm{min}$. The MS parameters are shown in SI 5Method 1 and 2. A $10 \mu \mathrm{L}$ sample was injected into the LC-MS/MS system.

\section{Preparation of OO-(HpODE)-TG isomer references}

Step I: Preparation of HpODE isomers. LA ( $2 \mathrm{~g})$ was dissolved in $50 \mathrm{~mL}$ of methanol. Rose bengal $(0.5 \mathrm{mg})$ was added, and the solution was exposed to light-emitting diode irradiation $\left(50,000\right.$ lux,$\left.>30^{\circ} \mathrm{C}\right)$ for $5 \mathrm{~h}$. Rose bengal was removed by Sep-Pak Vac QMA (3 cc, $500 \mathrm{mg}$, Waters, MA, USA), and the solution was evaporated under a nitrogen gas stream. The residue was dissolved in $50 \mathrm{~mL}$ of hexane, and the sample $(1 \mathrm{~mL})$ was injected into a semipreparative HPLC system (Shimadzu, Kyoto, Japan) to isolate the HpODE isomers. Inertsil SIL-100A $(5 \mu \mathrm{m}, 10 \times 250 \mathrm{~mm}$, GL Sciences Inc., Tokyo, Japan) was eluted with hexane/2-propanol/acetic acid (100:1:0.1, v/ $\mathrm{v} / \mathrm{v}$ ) at $20 \mathrm{~mL} / \mathrm{min} .{ }^{43}$ The column temperature was maintained at $40^{\circ} \mathrm{C}$, and the HpODE isomers were detected by UV absorbance at $210 \mathrm{~nm}$. To refine the purity of the references, the obtained HpODE isomers were subjected once more to semipreparative HPLC under the same conditions.

Step II: Esterification of HpODE and diacylglycerol. The hydroperoxide group of the purified HpODE isomer references were protected with MxP (ref. 41). Briefly, each HpODE isomer was dissolved in acetonitrile (44 mM), to which $1.8 \mathrm{mM}$ PPTS and $480 \mathrm{mM}$ MxP were added. After $1 \mathrm{~h}$, the reaction was stopped by adding an equivalent volume (to the reaction mixture) of 
water/methanol (4:1, v/v). The solution was loaded on a Sep-Pak Vac C18 (20 cc, $5 \mathrm{~g}$, Waters, MA, USA) equilibrated with methanol/water (3:7, v/v). The column was washed with $40 \mathrm{~mL}$ of methanol/water $(3: 7, \mathrm{v} / \mathrm{v})$, and the protected HpODE (HpODE-MxP) was eluted with $40 \mathrm{~mL}$ of methanol. The cis-trans isomers of 9-HpODE-MxP (i.e., 9-EZ-HpODE-MxP and 9-EE-HpODE$\mathrm{MxP}$ ), were separated by semipreparative RP-HPLC. An ODS column (Inertsil ODS-3, $10 \mu \mathrm{m}, 20 \times 250 \mathrm{~mm}$, GL Sciences Inc., Tokyo, Japan) was eluted with methanol/water/acetic acid (10:2:0.01, v/v/v). The obtained six HpODE-MxP isomers $(72 \mathrm{mM})$ were esterified with dioleoyl glycerol (78 $\mathrm{mM})$ in chloroform containing DCC (74 mM) and DMAP (82 mM) under nitrogen gas stream for $1.5 \mathrm{~h}$ at $22^{\circ} \mathrm{C} .{ }^{39}$ The synthesized OO-(HpODE-MxP)TG isomers were purified by semipreparative HPLC. Inertsil ODS-3 $(10 \mu \mathrm{m}$, $20 \times 250 \mathrm{~mm})$ was eluted with methanol/2-propanol $(3: 2, \mathrm{v} / \mathrm{v})$. The obtained OO-(HpODE-MxP)-TG isomers were deprotected as described previously. ${ }^{41}$ The resultant OO-HpODE-TG isomers (i.e., OO-(9-EZ-HpODE)TG and OO-(9-EE-HpODE)-TG, OO-(10-HpODE)-TG, OO-(12-HpODE)-TG, OO(13-ZE-HpODE)-TG and OO-(13-EE-HpODE)-TG) were chromatographically purified by semipreparative HPLC, using Inertsil ODS-3 $(10 \mu \mathrm{m}, 20 \times 250$ $\mathrm{mm}$ ) eluted with methanol/2-propanol $(3: 2, \mathrm{v} / \mathrm{v})$. OO-HpODE-TG $s n$-isomers was lastly separated with Inertsil SIL-100A (5 $\mu \mathrm{m}, 10 \times 250 \mathrm{~mm})$. Hexane/2propanol $(100: 0.3, \mathrm{v} / \mathrm{v})$ was used as mobile phase. The concentration of each reference was determined by measuring the concentration of $O A$, using the aforementioned GC method.

\section{Hydrolysis of OO-HpODE-TG references by a-lipase}

The hydroperoxide group of each OO-HpODE-TG reference $(45.7 \mu \mathrm{g})$ was protected with MxP as described above. The protected OO-HPODE-TG was dissolved in $0.1 \mathrm{~mL}$ of hexane. To the solution, $1 \mathrm{~mL}$ of a-lipase solution (10 $\mathrm{mg} / \mathrm{mL}$ in $50 \mathrm{mM}$ phosphate buffer (pH 6.8)) was added and the mixture was mixed vigorously at $37^{\circ} \mathrm{C}$ for $10 \mathrm{~min}$. The hydrolysate was extracted with $1 \mathrm{~mL}$ of hexane containing $0.1 \%(\mathrm{v} / \mathrm{v})$ acetic acid, evaporated under nitrogen gas and dissolved in $500 \mu \mathrm{L}$ of methanol. A portion of the sample $(5 \mu \mathrm{L})$ was analyzed by LC-MS. The samples were analyzed using an ODS column (Inertsil SIL-100A, $5 \mu \mathrm{m}, 2.1 \times 100 \mathrm{~mm}$, GL Sciences Inc., Tokyo, Japan) with a binary gradient consisting of solvent $A(0.1 \mathrm{mM}$ sodium acetate aqueous solution) and solvent $B$ (methanol containing $0.1 \mathrm{mM}$ sodium acetate). The gradient profile was as follows: $0-20 \mathrm{~min}, 50-70 \% \mathrm{~B}$ linear. The flow rate was $1.0 \mathrm{~mL} / \mathrm{min}$, and the column temperature was $40^{\circ} \mathrm{C}$. The MS parameters are shown in SI 5-Method 3 .

\section{MS/MS and LC-MS/MS analysis of OO-HpODE-TG isomers}

OO-HpODE-TG isomer references $(1 \mu \mathrm{M})$ were dissolved in methanol/2propanol $(100: 1, \mathrm{v} / \mathrm{v})$ containing $0.1 \mathrm{mM}$ sodium acetate. The samples were directly infused into 4000 QTRAP for Q1 mass- and product ion scan analysis. The MS/MS parameters are shown in SI 5-Method 4 and 5.

LC-MS/MS MRM analyses of oil samples were performed with Inertsil SIL-100A ( $5 \mu \mathrm{m}, 4.6 \times 250 \mathrm{~mm}$, GL Sciences Inc., Tokyo, Japan). Hexane/2propanol $(100: 0.3, \mathrm{v} / \mathrm{v})$ was used as the mobile phase. A flow gradient was used as follows: $0-9 \mathrm{~min}$; total flow $3-1 \mathrm{~mL} / \mathrm{min}$. The column was washed with methanol/2-propanol $(1: 1, \mathrm{v} / \mathrm{v})$ for $1 \mathrm{~h}$ once per 10 injections. The column oven temperature was maintained at $40^{\circ} \mathrm{C}$. The column eluent was mixed with a post-column solvent consisting of methanol/2-propanol (1:1, $\mathrm{v} / \mathrm{v}$ ) containing $0.3 \mathrm{mM}$ sodium acetate, at $0.4 \mathrm{~mL} / \mathrm{min}$. The combined flow was split at the post-column, and one of the split eluents was sent to the MS/MS system at $0.2 \mathrm{~mL} / \mathrm{min}$. OO-HpODE-TG isomers were detected using the MRM mode (SI 5-Method 6).

\section{Thermal- and photo- oxidation of canola oil}

For thermal-oxidation, fresh canola oil $(300 \mathrm{~mL})$ was put into an amber 500 $\mathrm{mL}$ glass beaker, and oxidized under gentle stirring at 25, 100, 140 and 180 ${ }^{\circ} \mathrm{C}$ in the dark. For photo-oxidation, oil (300 mL) was put into a clear $500 \mathrm{~mL}$ glass beaker, and gently stirred under photo-irradiation $(1000,10,000$ and 100,000 lux) at $25^{\circ} \mathrm{C}$. A portion of the oils ( $2 \mathrm{~mL}$ ) were collected at $10 \mathrm{~min}$ intervals for $2 \mathrm{~h}(n=3)$. The collected oils were 100 -fold diluted with hexane, and a portion $(50 \mu \mathrm{L})$ was analyzed using LC-MS/MS as described above in the MS/MS and LC-MS/MS analysis of OO/HpODE-TG isomers section.

\section{Peroxide value measurement}

POV was measured according to the official method of the American Oil Chemists' Society (AOCS) with slight modifications. ${ }^{22}$ Oxidized oil $(1 \mathrm{~g})$ was mixed with $5 \mathrm{~mL}$ of chloroform/acetic acid $(2: 3, \mathrm{v} / \mathrm{v})$. To the mixture, saturated potassium iodide solution $(0.1 \mathrm{~mL})$ was added and gently mixed for $1 \mathrm{~min}$. After the mixture was kept in the dark for $5 \mathrm{~min}$, distilled water $(5 \mathrm{~mL})$ and $1 \%(w t \%)$ starch solution $(0.1 \mathrm{~mL})$ were added. The mixture was centrifuged $\left(1000 \times g, 5 \mathrm{~min}, 4^{\circ} \mathrm{C}\right)$, and the lower layer was then removed. POV was calculated by titration with $10 \mathrm{mM}$ sodium thiosulfate in units of $10 \mu \mathrm{L}$.

\section{ACKNOWLEDGEMENTS}

This work was supported in part by Grant-in-Aid for Scientific Research (B) Grant Number $15 \mathrm{H} 04497$.

\section{AUTHOR CONTRIBUTIONS}

Study design-S.K., J.I., F.K., M.S., T.S., T.M., K.N. Experimental work-S.K., Y.H., Y.O., J.I., F.K. Data analysis-S.K. Writing manuscript-S.K., N.S., Y.O., S.T., T.E., K.N.

\section{ADDITIONAL INFORMATION}

Supplementary information accompanies the paper on the npj Science of Food website (https://doi.org/10.1038/s41538-017-0009-x).

Competing interests: The authors declare that they have no competing financial interests.

Publisher's note: Springer Nature remains neutral with regard to jurisdictional claims in published maps and institutional affiliations.

\section{REFERENCES}

1. Frankel, N. E. Chemistry of free radical and singlet oxidation of lipids. Prog. Lipid. Res. 23, 197-221 (1985).

2. Yoshida, Y., Umeno, A. \& Shichiri, M. Lipid peroxidation biomarkers for evaluating oxidative stress and assessing antioxidant capacity in vivo. J. Clin. Biochem. Nutr. 52, 9-16 (2013).

3. Goicoechea, E. \& Guillen, D. M. Analysis of hydroperoxides, aldehydes and epoxides by ${ }^{1} \mathrm{H}$ nuclear magnetic resonance in sunflower oil oxidized at 70 and $100^{\circ} \mathrm{C}$. J. Agric. Food. Chem. 58, 6234-6245 (2010).

4. Izaki, Y., Yoshikawa, S. \& Uchiyama, M. Effect of ingestion of thermally oxidized frying oil on peroxidative criteria in rats. Lipids 19, 324-331 (1984).

5. Halliwell, B. Free radicals, antioxidants, and human disease: curiosity, cause, or consequence? Lancet 344, 721-724 (1994).

6. Yang, M. C. et al. Thermally oxidized dietary fat and colon carcinogenesis in rodents. Nutr. Cancer 30, 69-73 (1998).

7. Jurek, D. et al. Dietary lipid hydroperoxides induce expression of vascular endothelial growth factor (VEGF) in human colorectal tumor cells. FASEB. J. https://doi.org/10.1096/fj.04-2111fje (2004).

8. Rohr-Udilova, V. N. et al. Lipid hydroperoxides from processed dietary oils enhance growth of hepatocarcinoma cells. Mol. Nutr. Food. Res. 52, 352-359 (2008).

9. Staprans, I., Pan, X.-M., Miller, M. \& Rapp, H. J. Effect of dietary lipid peroxides on metabolism of serum chylomicrons in rats. Am. J. Physiol. 264, G561-G568 (1993).

10. Staprans, I., Rapp, H. J., Pan, X.-M., Kim, Y. K. \& Feingold, R. K. Oxidized lipids in the diet are a source of oxidized lipid in chylomicrons of human serum. Arterioscler. Thromb. 14, 1900-1905 (1994).

11. Staprans, I., Rapp, H. J., Pan, X.-M. \& Feingold, R. K. Oxidized lipids in the diet are incorporated by the liver into very low density lipoprotein in rats. J. Lipid. Res. 37, 420-430 (1996).

12. Steinberg, D. Thematic review series: the pathogenesis of atherosclerosis. An interpretive history of the cholesterol controversy, part III: mechanistically defining the role of hyperlipidemia. J. Lipid. Res. 46, 2037-2051 (2005).

13. Asai, A. et al. Phosphatidylcholine hydroperoxide-induced THP-1 cell adhesion to intracellular adhesion molecule-1. J. Lipid. Res. 50, 957-965 (2009).

14. Asai, A. et al. Involvement of Rac GTPase activation in phosphatidylcholine hydroperoxide-induced THP-1 cell adhesion to ICAM-1. Biochem. Biophys. Res. Commun. 406, 273-277 (2011).

15. Kiko, T. et al. Significance of lutein in red blood cells of Alzheimer's disease patients. J. Alzheimers. Dis. 28, 593-600 (2012).

16. Kinoshita, M. et al. Age-related increases in plasma phosphatidylcholine hydroperoxide concentrations in control subjects and patients with hyperlipidemia. Clin. Chem. 46, 822-828 (2000).

17. Miyazawa, T., Suzuki, T. \& Fujimoto, K. Age-dependent accumulation of phosphatidylcholine hydroperoxide in the brain and liver of the rat. Lipids $\mathbf{2 8}, \mathbf{7 8 9 - 7 9 3}$ (1993). 
18. Dobarganes, C. M. \& Velasco, J. Analysis of lipid hydroperoxides. Eur. J. Lipid Sci. Technol. 104, 420-428 (2002).

19. Barriuso, B., Astiasaran, I. \& Ansorena, D. A review of analytical methods measuring lipid oxidation status in foods: a challenging task. Eur. Food Res. Technol. 236, 1-15 (2013).

20. Santas, J., Guzman, J. Y., Guardiola, F., Rafecas, M. \& Bou, R. High-throughput analysis of lipid hydroperoxides in edible oils and fats using the fluorescent reagent diphenyl-1-pyrenylphosphine. Food Chem. 162, 235-241 (2014).

21. Xu, L., Yu, X., Liu, L. \& Zhang, R. A novel method for qualitative analysis of edible oil oxidation using an electronic nose. Food Chem. 202, 229-235 (2016).

22. AOCS Official method Cd. "Peroxide value" 8-53 (1960).

23. Shantha, C. N. \& Decker, A. E. Rapid, sensitive, iron-based spectrophotometric methods for determination of peroxide values of food lipids. J. AOAC. Int. 77, 421-424 (1994).

24. Akasaka, K., Ohrui, H. \& Meguro, H. Simultaneous determination of hydroperoxides of phosphatidylcholine, cholesterol esters and triacylglycerols by columnswitching high-performance liquid chromatography with a post-column detection system. J. Chromatogr. 622, 153-159 (1993).

25. Miyazawa, T., Kunika, H., Fujimoto, K., Endo, Y. \& Kaneda, T. Chemiluminescence detection of mono-, bis, and tris- hydroperoxy triacylglycerols present in vegetable oils. Lipids. 30, 1001-1006 (1995).

26. Neff, E. W. \& Byrdwell, C. W. Characterization of model triacylglycerol (triolein, trilinolein and trilinolenin) autoxidation products via high-performance liquid chromatography coupled with atmospheric pressure chemical ionization mass spectrometry. J. Chromatogr. A. 818, 169-186 (1998).

27. Sugawara, T. \& Miyazawa, T. Microanalysis of triacylglycerol hydroperoxides by chemiluminescence-HPLC assay. Nihon yuka gakkaishi 48, 1391-1395 (1999). (Japanese).

28. Zeb, A. \& Murkovic, M. Carotenoids and triacylglycerols interactions during thermal oxidation of refined olive oil. Food. Chem. 127, 1584-1593 (2011).

29. Zeb, A. Triacylglycerols composition, oxidation and oxidation compounds in camellia oil using liquid chromatography-mass spectrometry. Chem. Phys. Lipids. 165, 608-614 (2012).

30. Zeb, A. Chemistry and liquid chromatography methods for the analyses of primary oxidation products of triacylglycerols. Free. Radic. Res. 49, 549-564 (2015).

31. Kato, S. et al. Liquid chromatography-tandem mass spectrometry determination of human plasma 1-palmitoyl-2-hydroperoxyoctadecadienoyl-phosphatidylcholine isomers via promotion of sodium adduct formation. Anal. Biochem. 471, 51-60 (2015).

32. Ito, J. et al. Direct separation of the diastereomers of phosphatidylcholine hydroperoxide bearing 13-hydroperoxy-9Z,11E-octadecadienoic acid using chiral stationary phase high-performance liquid chromatography. J. Chromatogr. A. 1386, 53-61 (2015)

33. Ito, J., Mizuochi, S., Nakagawa, K., Kato, S. \& Miyazawa, T. Tandem mass spectrometry analysis of linoleic and arachidonic acid hydroperoxides via promotion of alkali metal adduct formation. Anal. Chem. 87, 4980-4987 (2015).

34. Nakagawa, K., Kato, S. \& Miyazawa, T. Determination of phosphatidylcholine hydroperoxide $(\mathrm{PCOOH})$ as a marker of membrane lipid peroxidation. J. Nutr. Sci. Vitaminol. 61, 78-80 (2015).

35. Ito, J. et al. A novel chiral stationary phase HPLC-MS/MS method to discriminate between enzymatic oxidation and auto-oxidation of phosphatidylcholine. Anal. Bioanal. Chem. 408, 7785-7793 (2016).

36. Shimizu, N. et al. Mass spectrometric discrimination of squalene monohydroperoxide isomers. J. Oleo. Sci. 66, 227-234 (2017).
37. Kato, S. et al. Evaluation of the mechanisms of mayonnaise phospholipid oxidation. J. Oleo. Sci. 66, 369-374 (2017).

38. Howard, A. H. \& Ingold, U. K. Absolute rate constants for hydrocarbon autoxidation. VI. Alkyl aromatic and olefinic hydrocarbons. Can. J. Chem. 45, 793-802 (1967)

39. Lisa, M. \& Holcapek, M. Characterization of triacylglycerol enantiomers using chiral HPLC/APCI-MS and synthesis of enantiomeric triacylglycerols. Anal. Chem. 85, 1852-1859 (2013).

40. Caponio, F. et al. Diacylglycerol isomers in extra virgin olive oil: effect of different storage conditions. Food Chem. 140, 772-776 (2013).

41. Kato, S. et al. Preparation of 13 or 9-hydroperoxy-9Z,11E $(9 \mathrm{E}, 11 \mathrm{E})$ or $10 \mathrm{E}, 12 \mathrm{Z}$ $(10 \mathrm{E}, 12 \mathrm{E})$-octadecadienoic phosphatidylcholine hydroperoxide. J. Oleo. Sci. 63 431-437 (2014).

42. Ohta, Y., Yamane, T. \& Shimizu, S. Inhibition and inactivation of lipase by fat peroxide in the course of batch and continuous glycerolyses of fat by lipase. Agric. Biol. Chem. 53, 1885-1890 (1989).

43. Kondo, Y., Kawai, Y., Miyazawa, T. \& Mizutani, J. Chemiluminescence-HPLC analysis of fatty acid hydroperoxide isomers. Biosci. Biotech. Biochem. 57, 1575-1576 (1993).

44. Wada, S. \& Koizumi, C. Influence of the position of unsaturated fatty acid esterified glycerol on the oxidation rate of triglyceride. J. Am. Oil. Chem. Soc. 60 1105-1109 (1983).

45. Wijesundera, $C$. et al. Docosahexaenoic acid is more stable to oxidation when located at the $s n-2$ position of triacylglycerol compared to sn-1(3). J. Am. Oil. Chem. Soc. 85, 543-548 (2008).

46. Sies, H. \& Stahl, W. Vitamin E and C, $\beta$-carotene, and other carotenoids as antioxidants. Am. J. Clin. Nutr. 62, 1315S-1321S (1995).

47. Bohm, F., Edge, R. \& Truscott, G. Interactions of dietary carotenoids with singlet oxygen $\left({ }^{1} \mathrm{O}_{2}\right)$ and free radicals: potential effects for human health. Acta. Biochim. Pol. 59, 27-30 (2012).

48. Christaki, E., Bonos, E., Giannenas, I. \& Florou-Paneri, P. Functional properties of carotenoids originating from algae. J. Sci. Food. Agric. 93, 5-11 (2013).

49. Mukai, K. et al. Development of a singlet oxygen absorption capacity (SOAC) assay method. Measurements of the SOAC values for carotenoids and a-tocopherol in an aqueous Triton X-100 micellar solution. J. Agric. Food. Chem. 65, 784-792 (2017)

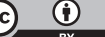

Open Access This article is licensed under a Creative Commons Attribution 4.0 International License, which permits use, sharing, adaptation, distribution and reproduction in any medium or format, as long as you give appropriate credit to the original author(s) and the source, provide a link to the Creative Commons license, and indicate if changes were made. The images or other third party material in this article are included in the article's Creative Commons license, unless indicated otherwise in a credit line to the material. If material is not included in the article's Creative Commons license and your intended use is not permitted by statutory regulation or exceeds the permitted use, you will need to obtain permission directly from the copyright holder. To view a copy of this license, visit http://creativecommons. org/licenses/by/4.0/.

(c) The Author(s) 2018 